\title{
FUNCTIONS OF INFINITELY MANY VARIABLES IN HILBERT SPACE*
}

\author{
BY
}

W. L. HART

1. Introduction. A point or vector $\xi=\left(x_{1}, x_{2}, \ldots, x_{i}, \ldots\right)$ with the denumerably infinite number of real coördinates $\left(x_{i}\right)$ is said to lie in Hilbert space if there exists for $\xi$ a convergent modulus $M \xi$ defined by the equation

$$
M \xi=\sqrt{\sum_{i=1}^{\infty} x_{i}^{2}}
$$

We shall denote this space by the abbreviation " $H$. " The present paper deals with theorems concerning real valued functions $f(\xi)$, defined for points $\xi$ in the region $R$ of $H$ determined by the condition $M \xi \leqq r$, in which $r$ is some positive number. The functions considered are in all cases assumed to be continuous according to a definition to be given in Section 2 . In dealing with continuous functions of infinitely many variables, the range selected for $\xi$ and the meaning assigned to the term "continuity" determine the nature of the hypotheses and proofs of the theorems which extend to infinitely many variables the various results found in the theory of functions of a real variable. In a previous paper $\dagger$ the author has considered functions $f(\xi)$ where $\xi$ had as its range the region $S$ defined by

$$
\left|x_{i}-a_{i}\right| \leqq s_{i} \quad(i=1,2, \ldots),
$$

where the $s_{i}$ were any positive mumbers. The continuity assumption made in Paper A was so strong and the region $S$ was of such a type that classical methods of proof with comparatively few additions sufficed for the development of a theory of real valued functions in $S$. In the present paper the range selected for $\xi$ and the weak continuity assumed for the function $f(\xi)$ are of such a nature that classical methods of proof must be radically modified before they can be applied.

The point of view of the discussion below is such that, as a consequence of the Riesz-Fischer $\ddagger$ Theorem concerning the Fourier constants of a summable

* Presented to the Society, September 8, 1920.

† These Tran s a c t i o n s, vol. 18 (1917), p. 125. This article will be referred to as Paper A.

$\ddagger$ Cf. Lalesco, Equations Intégrales, p. 95. 
function, the results obtained are capable of immediate application to problems in the theory of functionals. We shall consider the analogues for functions $f(\xi)$, defined for $\xi$ in $R$, of some of the most useful results in the theory of functions of a real variable. In Section 2 a mean value theorem is obtained from which there is derived a differential expression for $f(\xi)$ where the notion of a differential is defined in an appropriate manner. Hypotheses are then given under which an analogue of 'Taylor's theorem with an integral form for the remainder can be proved. In Section 3 the unique existence of a solution of an infinite system of ordinary differential equations is established. In Section 4 we shall consider the fundamental problem of implicit function theory for an infinite system of equations.

In Paper A results of the same character were obtained for the region $S$ but, because of the fundamental differences mentioned above between the present viewpoint and that of Paper $A$, there are no essential points of contact between the theorems for the region $S$ and those found below.

Functions in space $H$ were first considered by Hilbert in connection with the theory of integral equations. Numerous authors, following the road opened by Hilbert, have developed the theory of linear and bilinear forms in space $H$ together with their applications in the study of integral equations. In a recent article R. Gateaux* has considered some properties of functions $f(\xi)$ where $\xi$ had as its range a certain type of sub-space of the region. $H$. The present paper is not concerned with results of the type considered by Hilbert. A few of the results in Section 2 below are related to some theorems of Gateaux but no exact comparison is possible because of a fundamental difference between the region $R$ of this paper and the range for $\xi$ used by Gateaux. This difference will be referred to again in Section 2.

The notation used below is vectorial in character. Greek letters whenever used will denote vectors with infinitely many coördinates. Vector equations will imply the equality of the corresponding coördinates of the vectors represented by the two sides of the equality.

If $\left[\xi_{n}=\left(x_{1 n}, x_{2 n}, \ldots\right) ; n=1,2, \ldots\right]$ is a sequence of vectors, the limit vector, denoted by

$$
\alpha=\lim _{n \rightarrow \infty} \xi_{n} \quad\left(\alpha=a_{1}, a_{2}, \ldots\right),
$$

is defined by the equations

$$
a_{i}=\lim _{n \rightarrow \infty} x_{i n} \quad(i=1,2, \ldots)
$$

provided the limits exist. A Greek letter with an added notation will represent the vector obtained by adding the given notation to each coördinate of the

* Bulletin de la Société Mathématique de France, vol. 47 (1919), p. 70 . 
vector denoted by the unannotated letter. For example, if $\xi=\left(x_{1}, x_{2}, \ldots\right)$, then $\xi^{\prime}=\left(x_{1}^{\prime}, x_{2}^{\prime}, \ldots\right)$,

$$
\xi(t)=\left[x_{1}(t), x_{2}(t), \ldots\right], \frac{d \xi(t)}{d t}=\left[\frac{d x_{1}(t)}{d t}, \frac{d x_{2}(t)}{d t}, \ldots\right]
$$

If $\xi$ is in space $H$, this fact will be abbreviated by the notation " $\xi{ }^{H}$." The modulus of a vector in space $H$ will be denoted by prefixing $M$ to the vectorial symbol. For example, if $\xi$ and $\eta$ are points in $H$, the modulus and the square of the modulus of $(\xi-\eta)$ are represented, respectively, by

$$
M(\xi-\eta), \quad M^{2}(\xi-\eta) .
$$

When there is no possibility of confusion arising, a sequence of quantities will be represented by the type quantity enclosed in a bracket. For example, $\left(\xi_{n} ; n=1,2, \ldots\right)$ is abreviated by " $\left(\xi_{n}\right)$."

2. Theorems on continous functions. Let the function $f(\xi)$ be defined for a certain set $R$ of points $\xi$ in $H$. It will be convenient to use the following definition* of continuity:

DeFInIIION 1. The function $f(\xi)$ is continuous at a point $\alpha$ in $R$ if, whenever

$$
\lim _{n \rightarrow \infty} M\left(\xi_{n}-\alpha\right)=0,
$$

where $\xi_{n}$ is in $R$, it follows that

$$
\lim _{n \rightarrow \infty} f\left(\xi_{n}\right)=f(\alpha)
$$

If a sequence $\left(\xi_{n}\right)$ and a point $\alpha$ satisfy (1) it is said that the sequence converges to $\alpha$ in the strong sense. Continuity as here defined is of a much weaker type than complete continuity $\dagger$ which is frequently postulated for functions $f(\xi)$.

Consider in the future only those points $\xi^{H}$ in the region $R$ defined by the inequality $M \xi \leqq r$. If we consider the quantity $M\left(\xi_{n}-\alpha\right)$ as the distance ( $l^{\prime} e$ cart) of the two points $\xi_{n}$ and $\alpha$, the region $R$ is not $\ddagger$ compact. This fact constitutes the difference already referred to between the region $R$ and the region used by Gateaux. If a compact set of points were used instead of $R$ a different theory would result. Some of the theorems below which would remain true in a compact region could be proved under weaker hypotheses and the proofs in certain instances would be easier. However, from the point of view of the possible applications of the present results, it seems best to choose the region $R$ as specified above.

- Cr. Gateaux, loc. cit.

†Cf. Paper A, p. 129.

$\ddagger$ Cf. Gateaux, loc. cit., p. 72. 
For the region $R$ there is the well known analogue of the Weierstrass condensation theorem which is stated in

LEMMA* 1. If $\left(\xi_{n}\right)$ is a sequence of points in $R$ we can extract from it at least one sub-sequence $\left(\xi_{n}^{\prime}\right)$ to which there corresponds a point $\alpha$ in $R$ satisfying the equation

$$
\lim _{n \rightarrow \infty} \xi_{n}^{\prime}=\alpha .
$$

It should be noted that, as a consequence of the notational agreements of this paper, the convergence affirmed in the lemma requires merely that the coördinates $\left(x_{i_{n}}^{\prime}\right)$ of $\xi_{n}^{\prime}$ satisfy

$$
\lim _{n \rightarrow \infty} x_{i n}^{\prime}=a_{i} \quad\left(\alpha=a_{1}, a_{2}, \ldots ; i=1,2, \ldots\right) .
$$

Another well known property of the region $R$ is considered in

LEMMA 2. Suppose that $\left(\xi_{n}\right)$ is a sequence of points in $R$ which satisfies

$$
\lim _{n, m \rightarrow \infty} M\left(\xi_{n}-\xi_{m}\right)=0 .
$$

Then, there exists a point $\alpha$ in $R$ to which the sequence converges in the strong sense.

Because of its brevity the proof of this lemma will be repeated here. It is seen from (2) that, for every number $e>0$ there exists an integer $N_{\text {e such that }}$ if $n \geqq N_{\text {e }} m \geqq N_{e}$, then

$$
\sum_{i=1}^{k}\left(x_{i n}-x_{i m}\right)^{2} \leqq e \quad(k=1,2, \ldots) .
$$

It follows from (3) and from Lemma 1 that there exists a point $\alpha=\left(a_{1}, a_{2}, \ldots\right)$ in $R$ such that

$$
\lim _{n \rightarrow \infty} \xi_{n}=\alpha .
$$

Moreover, if $m$ in (3) approaches infinity while $n$ remains fixed, it is seen that we obtain the inequalities

$$
\sum_{i=1}^{k}\left(x_{i \pi}-a_{i}\right)^{2} \leqq e \quad(k=1,2, \ldots) .
$$

Hence, if $n \geqq N_{e}$, it follows from (4) that $M^{2}\left(\xi_{n}-\alpha\right) \leqq e$, which shows that equation (1) holds for $\alpha$ and the sequence $\left(\xi_{n}\right)$.

It should be noted that a function $f(\xi)$, which is continuous in $R$, does not necessarily attain its bounds if it is bounded in $R$. One property of ordinary continuous functions of a real variable which does persist for $f(\xi)$ is given in the obvious

\footnotetext{
- Cf. Riesz, loc. cit., p. 57.
} 
Proposirion 1. If $f(\xi)$ is continuous at a point $\alpha$ in $R$, it follows that for every number $e>0$ a number $d_{e}>0$ can be found such that if $M(\xi-\alpha) \leqq d_{e}$, then

$$
|f(\xi)-f(\alpha)| \leqq e .
$$

It will be useful later to have

Propositron 2. Suppose that $f(\xi)$ is continuous at all points in $R$. Then, for every $\xi$ in $R$, the function of $u$ represented by $f[\xi+u(\alpha-\xi)]$, where $\alpha$ is in $R$ and $0 \leqq u \leqq 1$, is continuous with respect to $\alpha$, uniformly for all values of $u$. That is, if

$$
\lim _{n \rightarrow \infty} M\left(\alpha_{n}-\alpha_{0}\right)=0
$$

then

$$
\lim _{n \rightarrow \infty} f\left[\xi+u\left(\alpha_{n}-\xi\right)\right]=f\left[\xi+u\left(\alpha_{0}-\xi\right)\right]
$$

uniformly for all values of $u$.

Let $\eta_{n}(u)=\xi+u\left(\alpha_{n}-\xi\right)$ and suppose that the uniformity specified in (6) does not hold. Then there must exist some number $e>0$ such that, to every integer $n$ there corresponds a value $u_{n}$ and an integer $m^{\prime} \geqq n$ such that

$$
\left|f\left[\eta_{m}^{\prime}\left(u_{n}\right)\right]-f\left[\eta_{0}\left(u_{n}\right)\right]\right|>e .
$$

In (7) it is seen that each $u_{n}$ is associated with a vector $\alpha_{m}$. Since all values $\left(u_{n}\right)$ lie on $0 \leqq u \leqq 1$, it follows that we can select a sub-sequence $\left(u_{n}^{\prime}\right)$, converging to a value $\bar{u}$. I,et $\left(\alpha_{n}^{\prime}\right)$ be the sub-sequence of $\left(\alpha_{n}\right)$ which is associated with the sequence $\left(u_{n}^{\prime}\right)$. Then, from (7) we obtain

(8) $\left|f\left[\eta_{n}^{\prime}\left(u_{n}^{\prime}\right)\right]-f\left[\eta_{0}\left(u_{n}^{\prime}\right)\right]\right|>e \quad\left(\eta_{n}^{\prime}\left(u_{n}^{\prime}\right)=\xi+u_{n}^{\prime}\left(\alpha_{n}^{\prime}-\xi\right) ; n=1,2, \ldots\right)$.

Since $\eta_{0}(\bar{u})=\xi+\bar{u}\left(\alpha_{0}-\xi\right)$, it follows that

$$
\begin{aligned}
M\left[\eta_{0}\left(u_{n}^{\prime}\right)-\right. & \left.\eta_{0}(\bar{u})\right]=\left|\bar{u}-u_{n}^{\prime}\right| M\left(\xi-\alpha_{0}\right), \\
M\left[\eta_{n}^{\prime}\left(u_{n}^{\prime}\right)-\eta_{0}(\bar{u})\right] & =M\left[\left(\bar{u}-u_{n}^{\prime}\right)\left(\xi-\alpha_{0}\right)+u_{n}^{\prime}\left(\alpha_{n}^{\prime}-\alpha_{0}\right)\right], \\
& \leqq\left|\bar{u}-u_{n}^{\prime}\right| M\left(\xi-\alpha_{0}\right)+u_{n}^{\prime} M\left(\alpha_{n}^{\prime}-\alpha_{0}\right) .
\end{aligned}
$$

The expressions on the right sides of (9) and (10) both approach zero as $n$ approaches infinity, so that, as a result of the continuity of $f$, it is seen that

$$
\lim _{n \rightarrow \infty} f\left[\eta_{n}^{\prime}\left(u_{n}^{\prime}\right)\right]=\lim _{n \rightarrow \infty} f\left[\eta_{0}\left(u_{n}^{\prime}\right)\right]=f\left[\eta_{0}(\bar{u})\right],
$$

which contradicts (8) if $n$ is sufficiently large. Thus the proposition has been established. 
As another obvious property of continuous functions we may state that if $f_{i}(\xi)(i=1,2, \ldots)$ are continuous in $R$ and if the series $S(\xi)=\Sigma f_{i}(\xi)$ converges uniformly for all $\xi$ in $R$, then $S(\xi)$ is continuous in $R$.

For a function $f(\xi)$ defined in $R$ we shall establish an analogue of the mean value theorem in

THEOREM I. Let $f(\xi)$ be continuous at all points in $R$. Suppose that there exist partial derivatives $\partial f(\xi) / \partial x_{j}(j=1,2, \ldots)$ which are continuous for $\xi$ in $R$ and are such that, for all $\xi$ in $R$, there exists

$$
\sum_{i=1}^{\infty}\left|\frac{\partial f(\xi)}{\partial x_{i}}\right|^{2} \leqq K
$$

where $K$ is some positive number. Then, for every pair of points $\left(\xi, \xi^{\prime}\right)$ in $R$, it follows that

$$
f\left(\xi^{\prime}\right)=f(\xi)+\sum_{i=1}^{\infty}\left(x_{i}^{\prime}-x_{i}\right) \int_{0}^{1} \frac{\partial f\left[\xi+u\left(\xi^{\prime}-\xi\right)\right]}{\partial x_{i}} d u .
$$

In order to prove the theorem let us first suppose that the two points $\left(\xi, \xi^{\prime}\right)$ satisfy

$$
M \xi<r, \quad M \xi^{\prime}<r .
$$

We notice that, for all values of $u$ on $0 \leqq u \leqq 1$, the point $\left[\xi+u\left(\xi^{\prime}-\xi\right)\right]$ is in $R$ because

$$
M\left[\xi+u\left(\xi^{\prime}-\xi\right)\right] \leqq(1-u) M \xi+u M \xi^{\prime}<r .
$$

Let $\zeta_{n}=\left(x_{1}^{\prime}, \ldots, x_{n}^{\prime}, x_{n+1}, \ldots\right)$. Then, since $\zeta_{n}$ differs from $\xi^{\prime}$ only in the coördinates after the $n$ th, it is seen that $M \zeta_{n}<r$ if $n$ is sufficientlylarge, and that

$$
\lim _{n \rightarrow \infty} M\left(\zeta_{n}-\xi^{\prime}\right)=0 .
$$

Hence

$$
\lim _{n \rightarrow \infty} f\left(\zeta_{n}\right)=f\left(\xi^{\prime}\right)
$$

If $n$ is so large that $M \zeta_{n}<r$, so that $\zeta_{n}$ is in the region $R$, it is easily established that the mean value theorem for the case of a finite number of variables can be applied to $f(\xi)$ with the result that

$$
f\left(\zeta_{n}\right)=f(\xi)+\sum_{i=1}^{n}\left(x_{i}^{\prime}-x_{i}\right) \int_{0}^{1} \frac{\partial f\left[\xi+u\left(\zeta_{n}-\xi\right)\right]}{\partial x_{i}} d u .
$$

Let us consider the function $S(\alpha)$ defined, for all $\alpha$ in $R$, by the equation

$$
S(\alpha)=\sum_{i=1}^{\infty}\left(x_{i}^{\prime}-x_{i}\right) \int_{0}^{1} \frac{\partial f[\xi+u(\alpha-\xi)]}{\partial x_{i}} d u .
$$


Let us first prove that $S(\alpha)$ converges uniformly for all $\alpha$ in $R$. As a consequence of the Lagrange-Cauchy* inequality for sums it is seen that the sum of the terms in (14) for $(i=n, \ldots, m)$ is, in absolute value, at most

$$
\sqrt{\sum_{i=n}^{m}\left(x_{i}^{\prime}-x_{i}\right)^{2}} \sqrt{\sum_{i=n}^{m}\left|\int_{0}^{1} \frac{\partial f[\xi+u(\alpha-\xi)]}{\partial x_{i}} d u\right|^{2}} \text {. }
$$

As a result of the Schwarz inequality for integrals,

$$
\left|\int_{a}^{b} f(x) g(x) d x\right|^{2} \leqq\left|\int_{a}^{b} f^{2}(x) d x\right| \cdot\left|\int_{a}^{b} g^{2}(x) d x\right|,
$$

it follows that (15) is at most equal to

$$
\sqrt{\sum_{i=n}^{m}\left(x_{i}^{\prime}-x_{i}\right)^{2}} \sqrt{\int_{0}^{1} \sum_{i=n}^{m}\left|\frac{\partial f[\xi+u(\alpha-\xi)]}{\partial x_{i}}\right|^{2}} d u \leqq \sqrt{K} \sqrt{\sum_{i=n}^{m}\left(x_{i}^{\prime}-x_{i}\right)^{2}},
$$

which, as $n$ and $m$ approach infinity, approaches zero uniformly for all $\alpha$ in $R$. Consequently $S(\alpha)$ converges uniformly.

In order to prove that $S(\alpha)$ is a continuous function of $\alpha$ it is necessary for us to show that each term in the series (14) is continuous. To do this we note that, if

$$
\lim _{n \rightarrow \infty} M\left(\alpha_{n}-\alpha\right)=0
$$

then, as a consequence of Proposition 2,

$$
\lim _{n \rightarrow \infty} \frac{\partial f\left[\xi+u\left(\alpha_{n}-\xi\right)\right]}{\partial x_{i}}=\frac{\partial f[\xi+u(\alpha-\xi)]}{\partial x_{i}},
$$

uniformly for all values of $u$ on $(0,1)$. From this it follows that

$$
\lim _{n \rightarrow \infty} \int_{0}^{1} \frac{\partial f\left[\xi+u\left(\alpha_{n}-\xi\right)\right]}{\partial x_{i}} d u=\int_{0}^{1} \frac{\partial f[\xi+u(\alpha-\xi)]}{\partial x_{i}} d u,
$$

which establishes the continuity of the terms in (14).

By a slight transformation of (13) we obtain

$$
f\left(\zeta_{n}\right)+\sum_{i=n+1}^{\infty}\left(x_{i}^{\prime}-x_{i}\right) \int_{0}^{1} \frac{\partial f\left[\xi+u\left(\zeta_{n}-\xi\right)\right]}{\partial x_{i}} d u=f(\xi)+S\left(\zeta_{n}\right)
$$

As a consequence of the continuity of the functions $S$ and $f$ it is seen that the limiting form of (17) as $n$ approaches infinity is

$$
f\left(\xi^{\prime}\right)=f(\xi)+S\left(\xi^{\prime}\right)
$$

\footnotetext{
- Riesz, Systèmes des Équations Linéaires, p. 36.
} 
which is the desired equation (12). It remains to show that (12) holds when $M \xi=r$ or $M \xi^{\prime}=r$. Suppose that one (or both) of these equalities has occurred, for example, $M \xi=r$. Let $\xi(v)$ represent the point whose coördinates $x_{i}(v)$ are defined by

$$
\begin{aligned}
& x_{i}(v)=x_{i} \\
& x_{i_{0}}(v)=x_{i_{0}}-v \frac{x_{i_{0}}}{x_{i_{0}}} \mid, \quad\left(i=1,2, \ldots ; i \neq i_{0}\right),
\end{aligned}
$$

where $i_{0}$ is some integer such that $x_{i_{0}} \neq 0$. For $v>0$, hut sufficiently small, $M \xi(v)<r$ and, for the same values of $v$, equation (12) is valid with $\xi$ replaced by $\xi(v)$. The right member of (12), after this replacement, is a continuous function of $v$ for $0 \leqq v \leqq 1$ which, as $v \rightarrow 0$, reduces to the right member of (12) as it is written in the statement of the theorem. The same procedure evidently applies if $M \xi^{\prime}=r$, so that the proof of the theorem is complete.

The proof of Theorem I would have been practically unchanged if, in place of condition (11), it had been assumed that the series

$$
\sum_{i=1}^{\infty}\left|y_{i} \frac{\partial f(\xi)}{\partial x_{i}}\right|
$$

converged uniformly for every pair of points $\xi$ and $\eta=\left(y_{1}, y_{2}, \ldots\right)$ in $R$.

Before considering the notion of the total differential of a function $f(\xi)$ let us first define a linear function $l(\xi)$ as one which satisfies the following conditions:

(a) $l(\xi)$ is continuous for all points $\xi^{H}$.

(b) For all points $\xi_{1}{ }^{H}$ and $\xi_{2}{ }^{H}, l\left(\xi_{1}+\xi_{2}\right)=l\left(\xi_{1}\right)+l\left(\xi_{2}\right)$.

In order to obtain an explicit expression for $l(\xi)$ let us consider those points in Hilbert space of the form $\left(x_{1}, \ldots, x_{n}, 0,0, \ldots\right)$, which we may denote by $(\xi)_{n}$. From conditions $(a)$ and $(b)$, as is well known, it follows that, if $\delta_{i}{ }^{H}$ is the point with all coördinates zero except the $i$ th, which is 1 , then

$$
l\left[(\xi)_{n}\right]=a_{1} x_{1}+\cdots+a_{n} x_{n} \quad\left(a_{i}=l\left(\delta_{i}\right)\right) .
$$

From condition (a) it is seen that

$$
l(\xi)=\lim _{n \rightarrow \infty} l\left[(\xi)_{n}\right]=\sum_{i=1}^{\infty} a_{i} x_{i}
$$

Since (18) converges for every $\xi^{H}$, it follows* that the point $\alpha=\left(a_{1}, a_{2}, \ldots\right)$ is itself in Hilbert space, and a function $l(\xi)$ is therefore identical with some linear form in infinitely many variables.

For a function $f(\xi)$ defined in $R$ let us define the notion of a differential as follows:

\footnotetext{
* Cf. Riesz, loc. cit., p. 47.
} 
Defrinirion 2. A function $f(\xi)$ has a differential $l\left(\xi, \xi^{\prime}\right)$ at a point $\xi$ of $R$ if $l\left(\xi, \xi^{\prime}\right)$ is a linear function of $\left(\xi^{\prime}-\xi\right)$ and if there exists a function $h\left(\xi, \xi^{\prime}\right)$ such that, for all $\xi^{\prime}$ of $R$,

$$
f\left(\xi^{\prime}\right)=f(\xi)+l\left(\xi, \xi^{\prime}\right)+h\left(\xi, \xi^{\prime}\right) M\left(\xi^{\prime}-\xi\right),
$$

where, moreover,

$$
\lim _{M\left(\xi^{\prime}-\xi\right) \rightarrow 0} h\left(\xi, \xi^{\prime}\right)=0 .
$$

With the aid of Theorem I let us establish

THEOREM II. Under the hypotheses of Theorem I with the condition (11) replaced by the assumption that the series in (11) converges uniformly for all $\xi$ in $R$, it follows that $f(\xi)$ possesses a differential $l\left(\xi, \xi^{\prime}\right)$. Moreover, the $l\left(\xi, \xi^{\prime}\right)$ and $h\left(\xi, \xi^{\prime}\right)$ corresponding to $f$ are continuous functions of $\left(\xi, \xi^{\prime}\right)$ at all points in $R$ and

$$
l\left(\xi, \xi^{\prime}\right)=\sum_{i=1}^{\infty}\left(x_{i}^{\prime}-x_{i}\right) \frac{\partial f(\xi)}{\partial x_{i}}
$$

As a consequence of equation (12) the $l\left(\xi, \xi^{\prime}\right)$ defined in (20) satisfies

$$
\begin{aligned}
& \left|f\left(\xi^{\prime}\right)-f(\xi)-l\left(\xi, \xi^{\prime}\right)\right|=\left|\sum_{i=1}^{\infty}\left(x_{i}^{\prime}-x_{i}\right) \int_{0}^{1}\left\{\frac{\partial f\left[\xi+u\left(\xi^{\prime}-\xi\right)\right]}{\partial x_{i}}-\frac{\partial f(\xi)}{\partial x_{i}}\right\} d u\right| \\
& \quad \leqq M\left(\xi^{\prime}-\xi\right) \sqrt{\sum_{i=1}^{\infty}\left(\int_{0}^{1}\left\{\frac{\partial f\left[\xi+u\left(\xi^{\prime}-\xi\right)\right]}{\partial x_{i}}-\frac{\partial f(\xi)}{\partial x_{i}}\right\} d u\right)^{2}}, \\
& \quad \leqq M\left(\xi^{\prime}-\xi\right) \sqrt{\int_{0}^{1} \sum_{i=1}^{\infty}\left(\frac{\partial f\left[\xi+u\left(\xi^{\prime}-\xi\right)\right]}{\partial x_{i}}-\frac{\partial f(\xi)}{\partial x_{i}}\right)^{2} d u}
\end{aligned}
$$

where the second inequality is a consequence of (16). In view of the type of convergence assumed for series (11), it is seen that the expression under the radical in (22) converges uniformly for all values of $\left(u, \xi, \xi^{\prime}\right)$. A course of reasoning similar to that used in regard to $S(\alpha)$ in (14) shows that the expression under the radical is a continuous function of $\xi^{\prime}$ and hence approaches zero if $\xi^{\prime}$ varies in such a way that $M\left(\xi^{\prime}-\xi\right) \rightarrow 0$.

Let us now define $h\left(\xi, \xi^{\prime}\right)$ by the equations

$$
\begin{aligned}
& h(\xi, \xi)=0, \\
& h\left(\xi, \xi^{\prime}\right) M\left(\xi^{\prime}-\xi\right)=f\left(\xi^{\prime}\right)-f(\xi)-l\left(\xi, \xi^{\prime}\right) \quad\left(\xi \neq \xi^{\prime}\right) .
\end{aligned}
$$

It is easily verified that the equation (19) is satisfied by $l$ and $h$ as defined in (20) and (23). Moreover, since $l\left(\xi, \xi^{\prime}\right)$ of equation (20) is continuous in $\left(\xi, \xi^{\prime}\right)$ for every pair of points in $R$, it follows from equation (19) that $h\left(\xi, \xi^{\prime}\right)$ possesses the same property. This completes the proof of Theorem II. 
By precisely the same method as was used in the proof of Theorem I we obtain in Theorem III below an analogue of Taylor's theorem for $f(\xi)$. The proof will not be given since, except for the greater complication of the formulas, the steps would be almost identical with those of the proof of Theorem I.

THEOREM III. Let $f(\xi)$ satisfy the hypotheses of Theorem I. Suppose that all partial derivatives used in the formulas below exist and are continuous for all points $\xi$ in $R$. Assume that the series

$$
\sum_{i_{1}, \ldots, i_{m}=1}^{\infty}\left|y_{i_{1}} \ldots y_{i_{m}} \frac{\partial^{(m)} f(\xi)}{\partial x_{i_{1}} \ldots \partial x_{i_{m}}}\right| \quad(m=1,2, \ldots, n,)
$$

converge uniformly for all points $\eta=\left(y_{1}, y_{2}, \ldots\right)$ and $\xi$ in $R$. Then, for every pair of points $\left(\xi, \xi^{\prime}\right)$ in $R$, it follow's that.

$$
\begin{gathered}
f\left(\xi^{\prime}\right)-f(\xi)=\sum_{i=1}^{\infty}\left(x_{i}{ }^{\prime}-x_{i}\right) \frac{\partial f(\xi)}{\partial x_{i}}+\sum_{i_{1}, i_{2}=1}^{\infty} \frac{\left(x_{i_{1}}^{\prime}-x_{i_{1}}\right)\left(x_{i_{2}}^{\prime}-x_{i_{2}}\right)}{2 !} \frac{\partial^{2} f(\xi)}{\partial x_{i_{1}} \partial x_{i_{2}}} \\
+\cdots+\sum_{i_{1}, \ldots, i_{n-1}=1}^{\infty} \frac{\left(x_{i_{1}}^{\prime}-x_{i_{1}}\right) \ldots\left(x_{i_{n-1}}^{\prime}-x_{i_{n-1}}\right)}{(n-1)} \frac{\partial^{(n-1)} f(\xi)}{\partial x_{i_{1}} \ldots \partial x_{i_{n-1}}} \\
+\sum_{i_{1}, \ldots, i_{n}=1}^{\infty} \frac{\left(x_{i_{1}}-x_{i_{1}}\right) \ldots\left(x_{i_{n}}^{\prime}-x_{i_{n}}\right)}{(n-1) !} \int_{0}^{1} \frac{\partial^{(n)} f\left[\xi+u\left(\xi^{\prime}-\xi\right)\right]}{\partial x_{i_{1}} \ldots x_{i_{n}}}(1-u)^{n-1} d u .
\end{gathered}
$$

3. Solution of an infinite System of differential equations. Consider the infinite system of ordinary differential equations

$$
\frac{d x_{i}}{d t}=f_{i}(t, \xi) \quad\left(\xi=\left|x_{1}, x_{2}, \ldots\right| ; i=1,2, \ldots\right)
$$

which in vector notation becomes

$$
\frac{d \xi}{d t}=\phi(t, \xi) \quad\left(\phi=f_{1}, f_{2}, \ldots\right) .
$$

Systems of the form (24) in which the $f_{i}$ were linear functions of $\xi$ entered as special cases in certain theories of E. H. Moore* and of T. H. Hildebrandt $\dagger$ in the domain of Moore's General Analysis. The author, also, has considered a theory of linear systems in which the right members of the equations were analytic functions of $t$. Certain of the linear systems included under the general theories of Moore and of Hildebrandt, as well as the linear type consid-

* Atti del IV. Congresso Internasionale dei Matematici (Roma, 1908), vol. 2, p. 98.

† These Tra n s a c tion s, vol. 18 (1917), p. 73.

† A merican J ournal of Mathematics, vol. 39 (1917), p. 407. 
ered previously by the author, when restricted to real values of the quantities entering, would satisfy the hypotheses imposed below on the system (24). In a course of lectures, which the author attended at the University of Chicago in 1915, E. H. Moore* established the existence of the solution of a differential equation of a general form in the field of General Analysis. The postulates of Moore, when interpreted for (24), differ in certain essential features from the hypotheses under which the system is considered in this paper.

In the consideration of (24) it will be convenient to denote by $W$ the class of all functions $\xi(t)$ with the following properties:

(a) For every value of $t$ on some interval $|t| \leqq T_{1}$ the function $\xi(t)$ is in $R$.

(b) If $\left|t^{\prime}\right| \leqq T_{1}$ and $\left|t^{\prime \prime}\right| \leqq T_{1}$, then

$$
\lim _{\left|t^{\prime}-t^{\prime \prime}\right| \rightarrow 0} M\left[\xi\left(t^{\prime}\right)-\xi\left(t^{\prime \prime}\right)\right]=0 .
$$

The existence of a solution of (24) will be established in

THEOREM IV. Let the functions $f_{i}$ in (24) be defined and continuous simultaneously in their arguments for $|t| \leqq T$, and $\xi$ in $R$. Assume that for every $(t, \xi)$ the modulus $M \phi(t, \xi)$ exists and that there is a number $A>0$ such that, for all $|t| \leqq \mathrm{T}$,

$$
M\left[\phi\left(t, \xi_{1}\right)-\phi\left(t, \xi_{2}\right)\right] \leqq A M\left(\xi_{1}-\xi_{2}\right),
$$

for every pair of points $\left(\xi_{1}, \xi_{2}\right)$ in $R$. Suppose, furthermore, that $M \phi(t, 0)$ has an upper bound $b>0$ for all $|t| \leqq T$. Then, if $|t|$ is sufficiently small there exists, among functions of the class $W$, one and only one function $\xi(t)$, satisfying (24) and the initial condition $\xi(0)=0$.

Let us first note that there exists a number $B>0$ such that, for all $(t, \xi)$,

$$
M \phi(t, \xi) \leqq B .
$$

This fact is a consequence of the inequality

$$
\begin{aligned}
M \phi(t, \xi) & \leqq M[\phi(t, \xi)-\phi(t, 0)]+M \phi(t, 0) \\
& \leqq A M \xi+M \phi(t, 0) \leqq A r+b,
\end{aligned}
$$

from which it follows that (26) is true with $B=A r+b$.

We also note that, if $\xi(t)$ is in the class $W$ for $|t| \leqq T_{1} \leqq T$, the expressions $f_{i}[t, \xi(t)]$ are continuous in $t$ because, if $\lim _{n \rightarrow \infty} t_{n}=i_{t}$, it follows that

$$
\lim _{n \rightarrow \infty} M\left[\xi\left(t_{n}\right)-\xi(\bar{t})\right]=0,
$$

so that

$$
\lim _{n \rightarrow \infty} f_{i}\left[t_{n}, \xi\left(t_{n}\right)\right]=f_{i}[\bar{t}, \xi(t)]
$$

* These lectures are unpublished. 
We shall show the existence of the solution $\xi(t)$ by proving that such a function is approached in the strong sense by the sequence $\left[\xi_{k}{ }^{(t)}\right]$ defined formally by the equations

$$
\begin{aligned}
\xi_{0} & =0 \\
\xi_{k}(t) & =\int_{0}^{t} \phi\left[t, \xi_{k-1}(t)\right] d t \quad(k=1,2, \ldots) .
\end{aligned}
$$

For the sake of simplicity in certain steps of the proof suppose that $t \geqq 0$; obvious modifications would suffice for the case $t<0$. In all integrals occurring below, the integration is with respect to $t$ and, for convenience, the symbol $d t$ will be omitted in the integrands. The convergence of the sequence $\left[\xi_{k}(t)\right]$ will be established in two steps. It will first be shown that a number $d>0$ exists so that for $|t| \leqq d$, the functions of (27) are defined and belong to the class $W$. It will then be shown that, for $|t| \leqq d$, the sequence (27) converges in the strong sense to a solution $\xi(t)$.

To accomplish the first step mentioned we shall show that there exists a number $d>0, d<T$, such that, for every number $e>0$ there can be found a number $g_{e}>0$ such that, for all values of $k$ and for all values of $\left(t, t^{\prime}\right)$ satisfying

$$
\left|t-t^{\prime}\right| \leqq g_{e},|t| \leqq d,\left|t^{\prime}\right| \leqq d,
$$

we have the inequalities.

$$
M\left[\xi_{k}(t)-\xi_{k}\left(t^{\prime}\right)\right] \leqq e, \quad M \xi_{k}(t) \leqq r .
$$

Consider (28) for $k=1$. With the aid of (16) it is easily verified that

$$
\begin{aligned}
\sum_{i=1}^{n}\left[\int_{t}^{t^{\prime}} f_{i}(t, 0)\right]^{2} & \leqq\left|t-t^{\prime}\right| \sum_{i=1}^{n}\left|\int_{t}^{t^{\prime}} f_{i}^{2}(t, 0)\right|=\left|t-t^{\prime}\right|\left|\int_{t}^{t^{\prime}} \sum_{i=1}^{n} f_{i}^{2}(t, 0)\right|, \\
& \leqq\left|t-t^{\prime}\right|\left|\int_{t}^{\prime \prime} M^{2} \phi(t, 0)\right| \leqq B^{2}\left|t-t^{\prime}\right|^{2}
\end{aligned}
$$

Since (29) is true for all values of $n$ it is seen that

$$
\left.M^{2}\left[\xi_{1}\left(t^{\prime}\right)-\xi_{1}(t)\right]\right]=\sum_{i=1}^{\infty}\left[\int_{t}^{t^{\prime}} f_{i}(t, 0)\right]^{2} \leqq B^{2}\left|t-t^{\prime}\right|^{2} .
$$

Let $d$ be the smaller of $T$ and $r / B$. Then it follows from (30) that (28) is true for $k=1$, so that $\xi_{1}(t)$ is a function of the class $W$. Consequently, the expressions $f_{i}\left[t, \xi_{1}(t)\right]$ are continuous in $t$ and therefore $\xi_{2}(t)$, as defined in (27), exists. The same procedure as in (29) and (30) now suffices to establish the truth of (28) for all values of $k$. The number $g_{e}$ noted above in connection with (28) can be selected independent of $k$ because the right member of the inequality corresponding to (30) would be the same for the function $\xi_{k}(t), k>1$, as it is for $\xi_{1}(t)$. 
To perform the second step of the proof let us first show by induction that

$$
M\left[\xi_{k}(t)-\xi_{k-1}(t)\right] \leqq \frac{A^{k-1} B t^{k}}{\sqrt{k !}} \quad(k=1,2, \ldots) .
$$

From the defining equations (27) and from (30) with $t^{\prime}=0$, we obtain the equations

$$
\begin{aligned}
M\left[\xi_{1}(t)-\xi_{0}\right] & \leqq B t, \\
M^{2}\left[\xi_{2}(t)-\xi_{1}(t)\right] & =M^{2}\left[\int_{0}^{t} \phi\left[t, \xi_{1}(t)\right]-\phi(t, 0)\right] \\
& =\sum_{i=1}^{\infty}\left[\int_{0}^{t} f_{i}\left[t, \xi_{1}(t)\right]-f_{i}(t, 0)\right]^{2} .
\end{aligned}
$$

As a consequence of (25) it is seen that

$$
\begin{aligned}
M^{2}\left[\phi\left[t, \xi_{1}(t)\right]-\phi(t, 0)\right] & =\sum_{i=1}^{\infty}\left[f_{i}\left[t, \xi_{1}(t)\right]-f_{i}(t, 0)\right]^{2} \leqq A^{2} M^{2}\left(\xi_{1}(t)-\xi_{0}\right) \\
& \leqq A^{2} B^{2} t^{2}
\end{aligned}
$$

Since, as a consequence of (16), the sum in (32) for $i=1, \ldots, n$ is at most

$$
\begin{aligned}
t\left(\sum_{i=1}^{n} \int_{0}^{t}\left\{f_{i}[t, \xi(t)]-f_{i}(t, 0)\right\}^{2}\right) & \left.=t \int_{0}^{t} \sum_{i=1}^{n}\left(f_{i} \mid t, \xi_{1}(t)\right]-f_{i}(t, 0)\right)^{2} \\
& \leqq t \frac{\left(A^{2} B^{2} t^{3}\right)}{3}
\end{aligned}
$$

it follows that

$$
M^{2}\left[\xi_{2}(t)-\xi_{1}(t)\right] \leqq \frac{t\left(A^{2} B^{2} t^{3}\right)}{3} \leqq \frac{A^{2} B^{2} t^{4}}{2 !}
$$

which establishes (31) for the case $k=2$. A simple induction proof, in which the steps would be the same as those taken in (32) to (34), would now suffice to establish (31) for all values of $k$.

As a consequence of $(31)$ for $k=m+1, \ldots, n$, it follows that

$$
M\left[\xi_{n}(t)-\xi_{m}(t)\right] \leqq \sum_{k=m+1}^{n} \frac{A^{k-1} B t^{k}}{\sqrt{k !}} \quad(n>m) .
$$

Since the series, whose general term is the expression under the summation sign in (35), converges uniformly for $|t| \leqq d$, it is seen that the left member of (35) approaches zero as $n$ and $m$ approach infinity, uniformly for $|t| \leqq d$. As a consequence of this uniformity it is readily established, by a method similar to that used in Lemma $2, \S 2$, that there exists a function $\xi(t)$ such that

$$
\lim _{n \rightarrow \infty} M\left[\xi_{n}(t)-\xi(t)\right]=0,
$$


uniformly for $|t| \leqq d$. Moreover, since the $g_{e}$ related to (28) was independent of $k$, it follows that

$$
\lim _{\left|t \rightarrow t^{\prime}\right| \rightarrow 0} M\left[\xi\left(t^{\prime}\right)-\xi(t)\right]=0,
$$

uniformly for all $|t| \leqq d,\left|t^{\prime}\right| \leqq d$. Thus $\xi(t)$ is a function of the class $W$.

To show that $\xi(t)$ is a solution of (24) it is first necessary to prove that, for every value of $i$,

$$
\lim _{n \rightarrow \infty} f_{i}\left[t, \xi_{n}(t)\right]=f_{i}[t, \xi(t)],
$$

uniformly for $|t| \leqq d$. Let us establish this by an indirect argument. Suppose, therefore, that the uniformity does not hold. It follows, by reasoning such as was used in Proposition 2, that a sequence of points $\left(t_{n}\right)$ can be found on the interval $|t| \leqq d$ with $\lim _{n \rightarrow \infty} t_{n}=t$ to which there corresponds a sub-sequence $\left\{\xi_{n}^{\prime}(t)\right\}$ of the functions of (27), satisfying the equations

$$
\left|f_{i}\left[t_{n}, \xi_{n}^{\prime}\left(t_{n}\right)\right]-f_{i}\left[t_{n}, \xi\left(t_{n}\right)\right]\right|>e \quad(n=1,2, \ldots) .
$$

In view of the fact that

$$
M\left[\xi^{\prime}{ }_{n}\left(t_{n}\right)-\xi\left(t_{n}\right)\right] \leqq M\left[\xi_{n}^{\prime}\left(t_{n}\right)-\xi_{n}^{\prime}(\bar{t})\right]+M\left[\xi^{\prime}{ }_{n}(\bar{t})-\xi(\bar{t})\right]+M\left[\xi(\bar{t})-\xi\left(t_{n}\right)\right],
$$

which approaches zero as $n$ approaches infinity, it follows from the continuity of $f_{i}(t, \xi)$ that the left side of (38) approaches zero as $n$ becomes infinite. Hence (38) has been contradicted and uniformity must exist in (37). Because of this uniformity it is seen that the limiting form of (27) as $k$ approaches infinity is

$$
\xi(t)=\int_{0}^{t} \phi[t, \xi(t)]
$$

from which (24) is obtained by differentiation.

It remains to prove that $\xi(t)$ is the unique solution of (24). Suppose there were a second solution $\eta(t)$ belonging to the class $W$ for $|t| \leqq d_{1} \leqq d$. Then $M[\xi(t)-\eta(t)] \leqq 2 r$. Moreover, we verify that

$$
\begin{aligned}
M^{2}[\xi(t)-\eta(t)] & =\sum_{i=1}^{\infty}\left\{\int_{0}^{t} f_{i}[t, \xi(t)]-f_{i}[t, \eta(t)]\right\}^{2} \\
& \leqq|t| \sum_{i=1}^{\infty}\left|\int_{0}^{t}\left\{f_{i}[t, \xi(t)]-f_{i}[t, \eta(t)]\right\}^{2}\right| \\
& \leqq|t|^{2}\left(4 r^{2} A^{2}\right),
\end{aligned}
$$

where the last inequality follows from (25) by the procedure illustrated in (34). In like manner we obtain in succession

$$
M^{2}[\xi(t)-\eta(t)] \leqq \frac{4 r^{2} A^{2 k}|t|^{2 k}}{k !} \quad(k=1,2, \ldots),
$$


from which it is seen that $\eta(t)=\xi(t)$ for $|t| \leqq d_{1}$. This completes the proof of Theorem IV.

With the assistance of Theorem I it is easy to establish

Corolinary 1. In place of condition (25), assume that there exist partial derivatives $\partial f_{i} / \partial x_{j}$ which are continuous in $(t, \xi)$ and are such that, for all $(t, \xi)$ satisfying $M \xi \leqq r,|t| \leqq T$, we have

$$
\left|\frac{\partial f_{i}(t, \xi)}{\partial x_{j}}\right| \leqq b_{i j}
$$

where $\sum_{i j=1}^{\infty} b_{i j}^{2}$ converges. Then, there exists a constant $A$ satisfying (25).

Because of Theorem I we may state that

$$
\begin{aligned}
\left|f_{i}\left(t, \xi_{1}\right)-f_{i}\left(t, \xi_{2}\right)\right|^{2} & =\left|\sum_{j=1}^{\infty}\left(x_{j_{2}}-x_{j_{1}}\right) \int_{0}^{1} \frac{\partial f_{i}\left[t, \xi_{1}+u\left(\xi_{2}-\xi_{1}\right)\right]}{\partial x_{j}} d u\right|^{2} \\
& \leqq M^{2}\left(\xi_{2}-\xi_{1}\right) \sum_{i j=1}^{\infty} b_{i j}^{2} .
\end{aligned}
$$

Hence (25) is satisfied with $A=\sqrt{\sum_{i j=1}^{\infty} b_{i j}^{2}}$.

In the next corollary there is established a characteristic of the convergence of the difference quotients $\Delta x_{i}(t) / \Delta t$ to the derivatives $d x_{i}(t) / d t$ which is useful in an application of Theorem IV to be treated in a later paper.

COROLLARY 2. In addition to the hypotheses of Theorem IV assume that there exists a number $C>0$ such that, for $\xi$ in $R$ and $\left|t_{1}\right| \leqq T,\left|t_{2}\right| \leqq T$,

$$
M\left[\phi\left(t_{1}, \xi\right)-\phi\left(t_{2}, \xi\right)\right] \leqq C\left|t_{1}-t_{2}\right| .
$$

Then, the function $\xi(t)$ satisfies the equation

$$
\lim _{\Delta \rightarrow 0} M\left[\frac{\xi(t+\Delta t)-\xi(t)}{\Delta t}-\phi[t, \xi(t)]\right]=0,
$$

uniformly for all values $|t| \leqq d$.

From equation (24) it is seen that, if $\left|t_{0}\right| \leqq d$,

$$
\frac{\xi\left(t_{0}+\Delta t\right)-\xi\left(t_{0}\right)}{\Delta t}-\phi\left[t_{0}, \xi\left(t_{0}\right)\right]=\int_{t_{0}}^{t_{0}+\Delta t}\left\{\frac{\phi[t, \xi(t)]-\phi\left[t_{0}, \xi\left(t_{0}\right)\right]}{\Delta t}\right\} .
$$

Call the quantity on the left $\eta\left(t_{0}, \Delta t\right)$. It is easily verified that

$$
(\Delta t)^{2} M^{2} \eta\left(t_{0}, \Delta t\right)=\sum_{i=i}^{\infty}\left[\int_{t_{0}}^{t_{0}+\Delta t}\left\{f_{i}[t, \xi(t)]-f_{i}\left[t_{0}, \xi\left(t_{0}\right)\right]\right\}\right]^{2} .
$$


The sum of the terms on the right in (41) for $i=1, \ldots, n$ is at most

$$
2|\Delta t|\left|\int_{t_{0}}^{t_{0}+\Delta t} \sum_{i=1}^{n}\left\{f_{i}[t, \xi(t)]-f_{i}\left[t_{0}, \xi(t)\right]\right\}^{2}+\left\{f_{i}\left[t_{0}, \xi(t)\right]-f_{i}\left[t_{0}, \xi\left(t_{0}\right)\right]\right\}^{2}\right|
$$

If $\Delta t$ is so small that, as a consequence of (36), $M^{2}\left[\xi(t)-\xi\left(t_{0}\right)\right] \leqq e$ for $t_{0} \leqq t$ $\leqq t_{0}+\Delta t$, it follows from (25), (39) and (42) that

$$
\begin{aligned}
\Delta t^{2} M^{2} \eta\left(t_{0}, \Delta t\right) & \leqq 2|\Delta t|\left|\int_{t_{0}}^{t_{0}+\Delta t}\left[C^{2}(\Delta t)^{2}+A^{2} e\right]\right| \\
& \leqq 2(\Delta t)^{2}\left[C^{2}(\Delta t)^{2}+A^{2} e\right] .
\end{aligned}
$$

Since $e$ in (43) can be selected arbitrarily small it follows that (40) holds for $t=t_{0}$. The uniformity affirmed in the corollary is a consequence of the fact that the steps in the reasoning above can be accomplished uniformly for all $\left|t_{0}\right| \leqq d$.

4. Implicit functions. Let us consider the solution of the infinite system of equations

$$
f_{i}(t, \xi)=0 \quad\left(\xi=x_{1}, x_{2}, \ldots ; i=1,2, \ldots\right),
$$

which, in the vector notation, become

$$
\phi(t, \xi)=0 \quad\left(\phi=f_{1}, f_{2}, \ldots\right),
$$

where $(t, \xi)$ are in the region $P$ defined by

$$
|t| \leqq T, \quad M \xi \leqq r .
$$

Suppose that $(t=0, \xi=0)$ satisfies (44). We shall establish the existence of a solution $\xi(t)$ for which $\xi(0)=0$, in

THEOREM V. In system (44) assume that the $f_{i}(t, \xi)$ are continuous in their arguments if $(t, \xi)$ are in $P$. Suppose that for all points in $P$ the series $\Sigma_{i=1}^{\infty} f_{i}^{2}(t, \xi)$ converges uniformly. Assume, moreover, that all derivatives $\partial f_{i}(t, \xi) / \partial x_{j}(i, j$ $=1,2, \ldots)$ exist and are continuous in $P$ and that the two series

$$
\sum_{i=1}^{\infty}\left|1-\frac{\partial f_{i}(t, \xi)}{\partial x_{i}}\right|, \quad \sum_{\substack{i, j=1 \\ i \neq j}}^{\infty}\left|\frac{\partial f_{i}(t, \xi)}{\partial x_{j}}\right|^{2},
$$

converge uniformly for $(t, \xi)$ in $P$. Then, if the convergent infinite determinant

$$
\Delta=\left|A_{i j}\right|_{i, j=1,2, \ldots} \neq 0 \quad\left[A_{i j}=\frac{\partial f_{i}(0,0)}{\partial x_{j}}\right],
$$

it follows that there exist positive numbers $c \leqq 1, d \leqq 1$ such that, for all points $t$ on the interval $|t| \leqq c T$, there corresponds one and only one point $\xi(t)$ in the region 
$M \xi \leqq d r$ such that $[t, \xi(t)]$ satisfies (44). All coördinates of the function $\xi(t)$ so determined are continuous for $|t| \leqq c T$.

The hypotheses of Theorem $\mathrm{V}$ as stated are stronger than is necessary and have been made so for convenience in the proof. At the end of the present section weaker hypotheses leading to the same conclusions will be mentioned. The theorem will be established by first obtaining a system

$$
\xi=\psi(t, \xi) \quad\left(\psi=h_{1}, h_{2}, \ldots\right),
$$

equivalent to (44) and by then solving (46) by the method of successive substitutions.

Define $\psi(t, \xi)$ as the function whose coördinates $h_{i}(t, \xi)$ are given by the solution of the infinite system of linear equations.

$$
f_{i}(t, \xi)+\sum_{j=1}^{\infty} A_{i j}\left(h_{j}-x_{j}\right)=0 \quad(i=1,2, \ldots) .
$$

Let $A_{i j}=d_{i j}+a_{i j}$ where $d_{i i}=1, d_{i j}=0(i \neq j)$. The infinite system (47) is of a type* for which the determinant $\Delta$ and all of its minors converge absolutely. Since we have assumed $\Delta \neq 0$, it follows $\dagger$ that there exists an infinite matrix

$$
B=\left(b_{i j}\right)_{i, j=1,2, \ldots}
$$

which is the reciprocal $\ddagger$ of the matrix $\left(-A_{i, j}\right)_{i i=1,2} \ldots$ and by means of which the solution of $(47)$ is given in the form

$$
h_{j}(t, \xi)-x_{j}=\sum_{k=1}^{\infty} b_{j k} f_{k}(t, \xi) \quad(j=1,2, \ldots) .
$$

Moreover, since $\Delta \neq 0$, the system (44) is equivalent $\S$ to the system (46) with $\psi(t, \xi)$ defined by $(47)$. After certain preliminary considerations we shall solve (46) by the method of successive substitutions.

Let us note some properties of the $b_{i j}$ which result from the special character of (47) and are consequences of the theorems proved in the sections of Equations Linéaires referred to in the preceding paragraph. Since, for every point $\alpha=$ $\left(a_{1}, a_{2}, \ldots\right)$ in space $H$, the system

$$
\sum_{j=1}^{\infty} A_{i j} z_{j}=a_{i} \quad(i=1,2, \ldots)
$$

* Riesz, loc. cit., p. 35.

$\dagger$ Riesz, loc. cit., $\$ 830,68,69$.

† Hellinger-Toeplitz, M a t h e $\mathrm{m}$ a $\mathrm{t}$ i s c h e A n n a 1 e n, vol. 69 (1910), p. 311. In the future, references to this article will be embodied in the text by the notation "H. $T$. p. (-)."

8 Cf. Riesz, loc. cit., p. 29. 
has a unique solution $\zeta=\left(z_{1}, z_{2}, \ldots\right)$ in $H$, where

$$
z_{j}=-\sum_{k=1}^{\infty} b_{j k} a_{k} \quad(j=1,2, \ldots),
$$

it follows (H.T. p. 321) that the matrix $B$ is limited in the Hilbert sense (H.T. p. 296). That is, there exists a bound $b>0$ such that, for all points $\xi^{\mathrm{B}}$ and $\eta^{\mathrm{B}}=$ $\left(y_{1}, y_{2}, \ldots\right)$,

$$
\left|\sum_{i, j=1}^{n} x_{i} b_{i j} y_{j}\right| \leqq b M \xi M \eta \quad(n=1,2, \ldots) .
$$

Since the matrix $B$ is limited with the bound $b$ it follows (H.T. p. 297) that each of the series

$$
\left(\sum_{k=1}^{\infty} b_{j k}^{2} ; j=1,2, \ldots\right),\left(\sum_{j=1}^{\infty} b_{j k}^{2} ; k=1,2, \ldots\right)
$$

converges and is at most $b^{2}$. Moreover, for every point $\alpha=\left(a_{1}, a_{2}, \ldots\right)$ in $H$; the point $\zeta$ whose coördinates $z_{j}$ are given by (50) satisfies (H.T. p. 301) the equation

$$
M \zeta \leqq b M a .
$$

On using (52) for the case of (47), we obtain

$$
M[\psi(t, \xi)-\xi] \leqq b M \phi(t, \xi) .
$$

It should be noted that, as a consequence of the present hypotheses, $M \phi(t, \xi)$ is a continuous function of $(t, \xi)$, and, moreover ${ }_{2} M \phi(0,0)=0$.

It will be necessary later to use the derivatives $\partial h_{i} / \partial x_{j}$ which, provided that the series used below converge uniformly, may be written

$$
\begin{aligned}
\frac{\partial h_{i}}{\partial x_{j}} & =d_{i j}+\sum_{k=1}^{\infty} b_{i k} \frac{\partial f_{k}(t, \xi)}{\partial x_{j}} \quad(i, j=1,2, \ldots), \\
\frac{\partial h_{i}}{\partial x_{j}} & =\sum_{k=1}^{\infty} b_{i k}\left(\frac{\partial f_{k}(t, \xi)}{\partial x_{j}}-A_{k i}\right) .
\end{aligned}
$$

The last reduction in (54) was a consequence of the equations

$$
d_{i j}=\sum_{k=1}^{\infty}-b_{i k} A_{k j} \quad(i, j=1,2, \ldots)
$$

which hold because $-B$ is the reciprocal of the matrix $\left(A_{i j}\right)$. The uniform convergence needed in writing (54) is established in

LEMMA 3. The infinite series

$$
S(t, \xi)=\sum_{i, k=1}^{\infty}\left|\frac{\partial f_{k}(t, \xi)}{\partial x_{i}}-A_{k i}\right|^{2}
$$


converges uniformly for all $(t, \xi)$ in $P$ and, as a consequence, the same fact can be stated concerning each of the series (54). Moreover, $S(t, \xi)$ is continuous if $(t, \xi)$ are in $P$.

By an obvious transformation of (55) we obtain the inequality

$$
S(t, \xi) \leqq 2 \sum_{i, k=1}^{\infty}\left|\frac{\partial f_{k}(t, \xi)}{\partial x_{i}}-d_{k i}\right|^{2}+2 \sum_{i, k=1}^{\infty}\left|d_{k i}-A_{k i}\right|^{2} .
$$

The uniform convergence of the series on the right, and hence the uniform convergence of $S(t, \xi)$, is implied by the type of convergence assumed for the series (45). The uniform convergence of (54) can be inferred from the inequality

$$
\left|\sum_{k=n}^{m} b_{i k}\left(\frac{\partial f_{k}(t, \xi)}{\partial x_{j}}-A_{k j}\right)\right|^{2} \leqq b^{2} \sum_{k=n}^{m}\left(\frac{\partial f_{k}(t, \xi)}{\partial x_{j}}-A_{k j}\right)^{2},
$$

which is obtained by use of the Lagrange-Cauchy inequality. When $n$ and $m$ approach infinity the right member of (56) approaches zero uniformly for $(t, \xi)$ in $P$ because cf the uniformity in (55). The continuity of $S(t, \xi)$ is evident because each term in the series (55) is continuous.

It is seen that $S(0,0)=0$. Hence, if a positive number $w<1$ is assigned, a positive number $d<1$ can be found so that $S(t, \xi) \leqq w^{2} / b^{2}$ if $(t, \xi)$ satisfy $M \xi$ $\leqq d r,|t| \leqq d T$.

With the aid of the properties possessed by $S(t, \xi)$, let us show that a positive number $c \leqq d$ can be selected so small that $c$ and $d$ satisfy the statement of Theorem V. In order to do this let us consider the sequence $\left[\xi_{k}(t)\right]$ defined by the equations

$$
\begin{aligned}
\xi_{0} & =0 \\
\xi_{k}(t) & =\psi\left[t, \xi_{k-1}(t)\right] \quad(k=1,2, \ldots) .
\end{aligned}
$$

We shall first prove that a number $c \leqq d$ can be found so that for all values $|t| \leqq$ $c T$ the sequence (57) is defined and satisfies $M \xi_{k}(t) \leqq d r$. As a consequence of (53) we obtain the inequality

$$
M \xi_{1}(t) \leqq b M \phi(t, 0) .
$$

Since $M \phi(0,0)=0$, we can select $c \leqq d$ so that, if $|t| \leqq c T$,

$$
M \xi_{1}(t) \leqq d r(1-w)<d r .
$$

By use of Theorem I we derive the equation

$$
\begin{aligned}
\xi_{2}(t)-\xi_{1}(t) & =\psi\left[t, \xi_{1}(t)\right]-\psi(t, 0) \\
& =\sum_{j=1}^{\infty} x_{j 1}(t) \int_{0}^{1} \frac{\partial \psi\left[t, \xi_{1}(t)+u\left(\xi_{1}(t)-\xi_{0}\right)\right]}{\partial x_{j}} d u .
\end{aligned}
$$


The coordinates of the derivatives $\partial \psi / \partial x_{j}$ are given by (54) and it is seen that the condition (11) of Theorem I is satisfied in the present case because, if $|t| \leqq c T$ and $M \xi \leqq d r$,

$$
\sum_{j=1}^{\infty}\left(\frac{\partial h_{i}(t, \xi)}{\partial x_{j}}\right)^{2} \leqq b^{2} S(t, \xi) \leqq \frac{b^{2} w^{2}}{b^{2}}=w^{2} \quad(i=1,2, \ldots) .
$$

LEMMA 4. If $|t| \leqq c T, M \xi^{\prime} \leqq d r, M \xi^{\prime \prime} \leqq d r$, the infinite matrix $K=\left(k_{i j}\right)$, whose elements are given by

$$
k_{i j}=\int_{0}^{1} \frac{\partial h_{i}\left[t, \xi^{\prime}+u\left(\xi^{\prime \prime}-\xi^{\prime}\right)\right]}{\partial x_{j}} d u \quad(i, j=1,2, \ldots),
$$

is limited and its bound may be taken as $w$.

First consider the matrix

$$
F=\left(\frac{\partial h_{i}(t, \xi)}{\partial x_{j}}\right)_{i, j=1,2, \ldots}
$$

From equation (54) it is seen that $F$ is the product of the matrices $B$ and $G$ where

$$
G=\left(\frac{\partial f_{k}(t, \xi)}{\partial x_{j}}-A_{k j}\right)_{k, j=1,2, \ldots}
$$

If $|t| \leqq c T$ and $M \xi \leqq d r$, it follows (H. T. p. 307) that $G$ is limited and that its bound may be taken as $\sqrt{S(t, \xi)} \leqq w / b$. Hence the matrix $F$ is limited and has the bound $b(w / b)=w($ H. T. p. 300). To show that $K$ has the bound $w$ it is merely necessary to note that, if $\alpha=\left(a_{1}, a_{2}, \ldots\right)$ and $\beta=\left(b_{1}, b_{2}, \ldots\right)$ are in Hilbert space then, on account of the limited character of $F$,

$$
\left|\sum_{i j=1}^{n} a_{i} k_{i j} b_{j}\right|=\left|\int_{0}^{1} \sum_{i j=1}^{n} a_{i} b_{j} \frac{\partial h_{i}\left[t, \xi^{\prime}+u\left(\xi^{\prime \prime}-\xi^{\prime}\right)\right]}{\partial x_{j}} d u\right| \leqq w M \alpha M \beta .
$$

From the definition of a limited matrix in (51) it is seen that (60) completes the proof of the lemma.

In order to establish Theorem V, return to equation (59). From Lemma 4 and from equation (52), interpreted for the matrix $K$ instead of $B$, it follows that

$$
M\left[\xi_{2}(t)-\xi_{1}(t)\right] \leqq w M \xi_{1}(t) \leqq w r d(1-w)<d r .
$$

Hence it is seen that

$$
M \xi_{2}(t) \leqq M\left[\xi_{2}(t)-\xi_{1}(t)\right]+M \xi_{1}(t) \leqq d r(1-w)(1+w)=d r\left(1-w^{2}\right) .
$$

In a similar manner it can be proved by induction that

$$
M\left[\xi_{k}(t)-\xi_{k-1}(t)\right] \leqq w M\left[\xi_{k-1}(t)-\xi_{k-2}(t)\right] \leqq w^{k-1} d r(1-w) .
$$


From equation (61) it is easily established by the method used in connection with (35) in Theorem IV, that there exists a function $\xi(t)$ such that

$$
\lim _{k \rightarrow \infty} M\left[\xi_{k}(t)-\xi(t)\right]=0,
$$

uniformly for $|t| \leqq c T$. On taking the limiting form of (57) as $k$ approaches infinity, we obtain

$$
\xi(t)=\psi[t, \xi(t)]
$$

and hence $\xi(t)$ is also a solution of (44).

The solution obtained is the only one existing for $|t| \leqq c T$ because, if for some value $\left|t_{1}\right| \leqq c T$ there was a second solution $\eta^{B}=\left(y_{1}, y_{2}, \ldots\right) \neq \xi\left(t_{1}\right)$ and satisfying $M \eta \leqq d r$, then

$$
\begin{aligned}
\xi\left(t_{1}\right)-\eta & =\psi\left[t_{1}, \xi\left(t_{1}\right)\right]-\psi\left(t_{1}, \eta\right) \\
& =\sum_{j=1}^{\infty}\left(x_{j}\left(t_{1}\right)-y_{j}\right) \int_{0}^{1} \frac{\partial \psi\left[t_{1}, \eta+u\left(\xi\left(t_{1}\right)-\eta\right)\right]}{\partial x_{j}} d u .
\end{aligned}
$$

From this it follows from Lemma 4 that

$$
M\left[\xi\left(t_{1}\right)-\eta\right] \leqq w M\left[\xi\left(t_{1}\right)-\eta\right],
$$

which is a contradictory statement unless $\xi\left(t_{1}\right)=\eta$. This completes the proof of Theorem V.

Let us consider how the hypotheses of Theorem $\mathrm{V}$ could be weakened. Instead of postulating the uniform convergence of $\sum_{i=1}^{\infty} f_{i}^{2}(t, \xi)$ it could be assumed that each function $f_{i}(t, \xi)$ has an upper bound $B_{i}>0$. We could then obtain a system, equivalent to (44) and satisfying the conditions of Theorem $V$, by dividing each function $f_{i}(t, \xi)$ by $i B_{i}$.

In the second place, by use of certain results from the theory of infinite determinants, * Theorem $\mathrm{V}$ could be proved with the first condition of (45) replaced by the assumption that

$$
\sum_{i=1}^{\infty}\left(1-\frac{\partial f_{i}(t, \xi)}{\partial x_{i}}\right)^{2}
$$

converges uniformiy.

It is not intended in the present paper to consider applications of the results that have been obtained. It should be noted, however, that the solution of the system of differential equations (24) suggests a means for the solution of a related type of differential-functional equations. The solution of the system (44) suggests a method for solving a related type of functional equations. The author will consider these applications in detail in a later paper.

* Cf. Riesz, loc. cit., p. 39.

UNIVERSITY OF MINNESOTA, MnNeapolis, MinN. 